Удк

\title{
МОДЕЛИРОВАНИЕ ЧАСТОТНО-СЕЛЕКТИВНЫХ ПОВЕРХНОСТЕЙ ДЛЯ МНОГОДИАПАЗОННЫХ И ДВУХПОЛЯРИЗАЦИОННЫХ ПРИМЕНЕНИЙ С ПОМОЩЬЮ ИТЕРАЦИОННОГО МЕТОДА ВОЛНОВОГО ПРЕДСТАВЛЕНИЯ
}

\author{
А. ДЖУЙМАА ${ }^{1}$, М. ТИТАУЙНЕ ${ }^{1,2}$, И. АДУИ ${ }^{2}$, Т. Р. ДЕ СОУЗА ${ }^{3}$, А. Г. НЕТО ${ }^{3}$, Г. БАДРО ${ }^{4}$ \\ ${ }^{1}$ Университет Батны, \\ Алжир, Батна, 05000 \\ ${ }^{2}$ Университет Башира эль-Ибрахима, \\ Алжир, Бордж-Бу-Арреридж, El Anasseur 34625 \\ ${ }^{3}$ Федеральный университет штата Параиба, \\ Бразилия, Жуан-Песоа, Параиба, СЕР 58015-430 \\ ${ }^{4}$ Университет Тулузы, \\ Франичия, Тулуза, ВР 7122, F-31071
}

\begin{abstract}
Аннотация. В статье предложена новая многодиапазонная двухполяризационная частотно-селективная поверхность FSS (frequency selective surface) для применения в обтекателях антенн скрытных радиолокаторов. Конструкция основана на структуре разомкнутого металлического кольца со связанными концами полосок. Предложенная FSS одновременно отражает электромагнитные волны на частотах 9,1, 10,8 и 11,5 ГГц с полосами отражения 900, 100 и 200 МГц, соответственно, при возбуждении структуры плоской волной, поляризованной вдоль оси $x$, и отражает на одной частоте 7,3 ГГц с полосой отражения 1000 МГц, если структура возбуждается плоской волной $y$-поляризации. Получение необходимых частотных характеристик обеспечивается за счет варьирования длины связанных полосок FSS и левого края вертикально ориентированной П-подобной металлической полоски, входящей в структуру FSS. Достигнуто расширение полос отражения при возбуждении FSS волной $x$-поляризации с 900 до 1500 МГц и с 200 и 100 до 500 МГц. Результаты моделирования, полученные итерационным методом волнового представления, сравнены с результатами моделирования, полученными с помощью программного обеспечения COMSOL multiphysics software $4.3 \mathrm{~b}$, и результатами измерений. Достигнуто хорошее согласование результатов.
\end{abstract}

Ключевые слова: FSS; итерационный метод волнового представления; разомкнутое металлическое кольцо со связанными концами полосок; вертикальная П-подобная металлическая полоска; обтекатели для скрытных радиолокаторов; многодиапазонный режим работы; двойная поляризация; частотная настройка; расширение полосы отражения

\section{1. ВВЕДЕНИЕ}

В последнее время возрастает потребность в частотно-селективных поверхностях FSS (frequency selective surface), имеющих небольшие размеры, многодиапазонный и двухполяризационный режимы работы [1-3]. Использование многочастотного и поляризационного разнесения уменьшает интерференционные помехи электромагнитных волн, что является необходимым в беспроводных системах связи $[4,5]$. Структуры на основе FSS широко применяются в системах связи, начиная со сверхвысокочастотных систем и антенн, и заканчивая радиолокаторами и спутниковыми системами связи $[6,7]$.

Одним из важных применений FSS являются гибридные обтекатели, используемые 


\section{БИБЛИОГРАФИЧЕСКИЙ СПИСОК}

1. Qiang T. A compact dual-wideband bandpass filter using two triple-mode resonators for S-band applications / Tian Qiang, Cong Wang, Nam Young Kim // Microw. Opt. Technol. Lett. - Jan. 2015. - Vol. 57, No. 1. - P. 153-157. - DOI : 10.1002/mop.28799.

2. Koley $S$. A planar microstrip-fed tri-band filtering antenna for WLAN/WiMAX applications / Santasri Koley, Debjani Mitra // Microw. Opt. Technol. Lett. — Jan. 2015. - Vol. 57, No. 1. - P. 233-237. — DOI : $10.1002 /$ mop. 28813.

3. Lim I. CPW-fed arbitrary frequency-switchable antenna using CRLH transmission line / Inseop Lim, Sungjoon Lim // ETRI J. - Feb. 2014. - Vol. 36, No. 1. - P. 151-154. - DOI : 10.4218/etrij.14.0213.0027.

4. Microstrip antenna using $\mathrm{H}$-slotted ground structure for orthogonally polarized dual-band operation / Haechul Choi, Eunsook Lim, Dong-Hyo Lee, Seongmin Pyo // Microw. Opt. Technol. Lett. - Jan. 2016. - Vol. 58, No. 1. - P. 136-139. - DOI : 10.1002/mop.29512.

5. An active ring slot with RF MEMS switchable radial stubs for reconfigurable frequency selective surface applications / R. Martinez-Lopez, J. Rodriguez-Cuevas, A. E. Martynyuk, J. I. Martinez-Lopez // PIER. - 2012. - Vol. 128. - P. 419-440. — DOI : $10.2528 /$ PIER12 041207.

6. Azemi S. N. A reconfigurable FSS using a spring resonator element / Saidatul Norlyana Azemi, Kamran Ghorbani, Wayne S. T. Rowe // IEEE Antennas Wireless Propag. Lett. - 2013. - Vol. 12. - P. 781-784. — DOI : 10.1109/LAWP.2013.2270950.

7. Li M. Liquid-tunable frequency selective surfaces / Meng Li, Bin Yu, Nader Behdad // IEEE Microwave
Wireless Compon. Lett. - Aug. 2010. - Vol. 20, No. 8. — P. 423-425. - DOI : 10.1109/LMWC.2010.2049257.

8. A FSS with stable performance under large incident angles / Tao Ma, Hang Zhou, Yuan Yang, Bo Liu // PIER Lett. - 2013. - Vol. 41. - P. 159-166. - DOI : 10.2528/PIERL13061703.

9. Filter-antenna consisting of conical FSS radome and monopole antenna / Hang Zhou, Shaobo Qu, Baoqin Lin, Jiafu Wang, Hua Ma, Zhuo Xu, Weidong Peng, Peng Bai // IEEE Trans. Antennas Propag. - Jun. 2012. Vol. 60, No. 6. - P. 3040-3045. - DOI : 10.1109/TAP. 2012.2194648.

10. Dual-polarized FSS with wide frequency tunability and simple bias network / Hang Zhou, Xinhua Wang, Shaobo Qu, Lin Zheng, Hangying Yuan, Mingbao Yan, Yongfeng Li, Jiafu Wang, Hua Ma, Zhuo Xu // Progress in Electromagnetics Research : Symp. PIERS, 25-28 Aug. 2014, Guangzhou, China : proc. - 2014.

11. Nachal S. L. Design of frequency selective structures for radio wave propagation / S. Lalitha Nachal, B. Monica, M. Ramani // Int. J. P2P Network Trends Technol. - Apr. 2014. - Vol. 7, No. 4. - P. 34-39. URL : http://www.ijpttjournal.org/archives/ijptt-v7p407.

12. Chang T. K. Frequency selective surfaces on biased ferrite substrates / T. K. Chang, R. J. Langley, E. A. Parker // Electron. Lett. — 1994. — Vol. 30, No. 15. — P. 1193-1194. — DOI : 10.1049/el:19940823.

13. Measurement of electromagnetic properties of liquid crystals at $300 \mathrm{GHz}$ using a tunable FSS / R. Simms, Raymond Dickie, Robert Cahill, Neil Mitchell, Harold Gamble, Vincent Fusco // Antennas for Space Applications : 31st ESA Antenna Workshop, 1 Oct. 2010, Noordwijk, Netherlands : proc. -2010.

14. Chang T. K. Active frequency-selective surfaces / T. K. Chang, R. J. Langley, E. A. Parker // IEE Proc. Microwaves, Antennas Propag. - Feb. 1996. - Vol. 143, No. 1. - P. 62-66. - DOI : 10.1049/ip-map:19960115.

15. Electromagnetic modeling of quasi-square open metallic ring frequency selective surface using wave concept iterative procedure / Mohammed Titaouine, Nathalie Raveu, Alfredo Gomes Neto, Henri Baudrand // ETRI J. — Feb. 2009. — Vol. 31, No. 1. - P. 77-79. — DOI : 10.4218 /etrij.09.0208.0268.

16. Efficient analysis of complex FSS structure using the WCIP method / Sassi Aroussi, Latrach Latrach, Noureddine Sboui, Ali Gharsallah, Abdelhafidh Gharbi, Henry Baudrand // J. Electromagnetic Analysis Applications. - 2011. - Vol. 3. - P. 447-451. — DOI : 10.4236/jemaa.2011.311071.

17. Analysis of frequency selective surface isotropic/anisotropic layers using WCIP method / Mohammed Titaouine, Alfredo Gomes Neto, Henri Baudrand, Farid Djahli // ETRI J. — Feb. 2007. - Vol. 29, No. 1. - P. 36-44. — DOI : 10.4218/etrij.07.0106.0123. 